\title{
Neutron spin rotation measurements
}

\author{
M. Sarsour ${ }^{1, a}$, J. Amadio ${ }^{2}$, E. Anderson ${ }^{3}$, L. Barrón-Palos 4 , B. Crawford ${ }^{2}$, C. Crawford ${ }^{5}$, D. Esposito ${ }^{6}$, W. Fox ${ }^{3}$, \\ I. Francis ${ }^{7}$, J. Fry ${ }^{8}$, H. Gardiner ${ }^{9}$, C. Haddock ${ }^{10}$, A. Holly ${ }^{11}$, S.F. Hoogerheide ${ }^{12}$, K. Korsak ${ }^{3}$, J. Lieers ${ }^{13}$, S. Magers ${ }^{2}$, \\ M. Maldonado-Velázquez ${ }^{4}$, D. Mayorov ${ }^{14}$, H.P. Mumm ${ }^{12}$, J.S. Nico ${ }^{12}$, T. Okudaira ${ }^{10}$, C. Paudel ${ }^{1}$, S. Santra ${ }^{15}$, \\ H.M. Shimizu ${ }^{10}$, W.M. Snow ${ }^{3}$, A. Sprow ${ }^{5}$, K. Steen ${ }^{3}$, H.E. Swanson ${ }^{16}$, F. Tôvesson ${ }^{14}$, J. Vanderwerp ${ }^{3}$, and P.A. Yergeau ${ }^{2}$ \\ ${ }^{1}$ Georgia State University, Atlanta, GA 30303, USA \\ ${ }^{2}$ Gettysburg College, Gettysburg, PA 17325, USA \\ ${ }^{3}$ Indiana University, Bloomington, IN 47408, USA \\ ${ }^{4}$ Universidad Nacional Autónoma de México, D.F. 04510, México \\ ${ }^{5}$ University of Kentucky, Lexington, KY 40506, USA \\ ${ }^{6}$ University of Dayton, Dayton, OH 45469, USA \\ ${ }^{7} 612$ S Mitchell St Bloomington, IN 47401, USA \\ ${ }^{8}$ University of Virginia, Charlottesville, VA 22903, USA \\ ${ }^{9}$ Louisiana State University, Baton Rouge, LA 70803, USA \\ ${ }^{10}$ Nagoya University, Furocho, Chikusa Ward, Nagoya, Aichi Prefecture 464-0814, Japan \\ ${ }^{11}$ Tennessee Tech University, Cookeville, TN 38505, USA \\ ${ }^{12}$ National Institute of Standards and Technology, Gaithersburg, MD 20899, USA \\ ${ }^{13}$ Embry-Riddle Aeronautical University, Daytona Beach, FL 32114, USA \\ ${ }^{14}$ Los Alamos National Lab, Los Alamos, NM 87545, USA \\ ${ }^{15}$ Bhabha Atomic Research Centre, Trombay, Mumbai, Maharashtra 400085, India \\ ${ }^{16}$ University of Washington, Seattle, WA 98105, USA
}

\begin{abstract}
The neutron spin rotation (NSR) collaboration used parity-violating spin rotation of transversely polarized neutrons transmitted through a $0.5 \mathrm{~m}$ liquid helium target to constrain weak coupling constants between nucleons. While consistent with theoretical expectation, the upper limit set by this measurement on the rotation angle is limited by statistical uncertainties. The NSR collaboration is preparing a new measurement to improve this statistically-limited result by about an order of magnitude. In addition to using the new high-flux NG-C beam at the NIST Center for Neutron Research, the apparatus was upgraded to take advantage of the larger-area and more divergent NG-C beam. Significant improvements are also being made to the cryogenic design. Details of these improvements and readiness of the upgraded apparatus are presented. We also comment on how recent theoretical work combining effective field theory techniques with the $1 / N_{c}$ expansion of QCD along with previous $\mathrm{NN}$ weak measurements can be used to make a prediction for $d \phi / d z$ in ${ }^{4} \mathrm{He}$.

An experiment using the same apparatus with a room-temperature target was carried out at LANSCE to place limits on parity-conserving rotations from possible fifth-force interactions to complement previous studies. We sought this interaction using a slow neutron polarimeter that passed transversely polarized slow neutrons by unpolarized slabs of material arranged so that this interaction would tilt the plane of polarization and develop a component along the neutron momentum. The results of this measurement and its impact on the neutron-matter coupling $g_{A}^{2}$ from such an interaction are presented. The NSR collaboration is also preparing a new measurement that uses an upgraded version of the room-temperature target to be run on the NG-C beamline; and it is expected to constrain $g_{A}^{2}$ by at least two additional orders of magnitude for $\lambda_{c}$ between $1 \mathrm{~cm}$ and $1 \mu \mathrm{m}$.
\end{abstract}

\section{Introduction}

The nucleon-nucleon $(\mathrm{NN})$ weak interaction is one of the most poorly understood areas of the Standard Model. Understanding the NN weak interaction in general and NN weak interaction amplitudes in particular is very important for several reasons [1-4]. It is a probe of Quantum Chromodynamics (QCD) that samples the shortdistance quark-quark correlations in $\mathrm{QCD}$ and its chiral

a e-mail: msar@gsu.edu symmetry-dominated long-range properties. Knowledge of weak NN couplings can shed light on parity violation phenomena in atomic and nuclear physics [5-8]. The theoretical advances in the description of NN weak interaction set the stage for eventual quantitative predictions of the weak interaction directly from the Standard Model and QCD and thus make better contact with QCD in the near future.

The uncertainty in details of the hadronic weak interaction (HWI) stems largely from the very short range of the quark-quark weak interaction $(\sim 0.01 \mathrm{fm})$ compared

(C) The Authors, published by EDP Sciences. This is an Open Access article distributed under the terms of the Creative Commons Attribution License 4.0 (http://creativecommons.org/licenses/by/4.0/). 
with the range of nucleon-nucleon interaction $(\sim 1 \mathrm{fm})$ and the relative strength of the weak to strong force between strongly interacting particles $\left(10^{-7}-10^{-6}\right)$. Experimentally one uses parity violation to isolate the weak contribution. The primary analysis tool over the past few decades has been the meson exchange model of Desplanques, Donoghue, and Holstein (DDH) [9, 10]. The DDH models the HWI between nucleons as an exchange of a light meson $(\pi, \rho, \omega)$ where the meson couples to the nucleons via strong coupling at one vertex and weak coupling at the other. This model leads to seven meson coupling constants, $h_{\pi}^{1}, h_{\omega}^{0,1}, h_{\rho}^{0,1,1^{\prime}, 2}$, which need to be fixed by measurement. Several measurements from NN system to heavy nuclei were carried out but only some place constraints on HWI [11], which include $\overrightarrow{\mathrm{p}}+\mathrm{p}[12-17], \overrightarrow{\mathrm{p}}+{ }^{4} \mathrm{He}[18,19]$, $P_{\gamma}\left({ }^{18} \mathrm{~F}\right)$ [20-23], and $A_{\gamma}\left({ }^{19} \mathrm{~F}\right)$ [24-26]. In heavy nuclei measurements, an asymmetry enhancement is observed but the theory calculations are complicated [5,27]. This difficulty motivated studying few body systems because while they are expected to have small asymmetries, $10^{-7}$ $10^{-8}$, the associated theory is calculable and reliable.

NPDGamma experiment is the first few nucleon precision measurement of $h_{\pi}^{1}$ with little or no theoretical ambiguity [28,29]. NPDGamma experiment measured the parity-violating asymmetry in polarized neutron capture on protons, $A_{\gamma}(\overrightarrow{\mathrm{n} p} \rightarrow \mathrm{d} \gamma)$, which is proportional to $h_{\pi}^{1}$. The experiment completed data collection and the final results show a small value for $h_{\pi}^{1}=[+2.6 \pm 1.2$ (stat.) \pm 0.2 (sys.)] $\times 10^{-7}$ [29]. Other few body measurements include neutron capture on ${ }^{3} \mathrm{He}\left(\overrightarrow{\mathrm{n}}+{ }^{3} \mathrm{He}\right)$ at the SNS and neutron spin rotation in ${ }^{4} \mathrm{He}\left(\overrightarrow{\mathrm{n}}+{ }^{4} \mathrm{He}\right)$ at NIST (the focus of this proceeding). Within the framework of the DDH model, the $\vec{n}+{ }^{4} \mathrm{He}$ is especially interesting. It is the isospin mirror of $\vec{p}+{ }^{4} \mathrm{He}$ and it would allow an isoscalar/isovector separation. The linear combination of $\mathrm{NN}$ weak amplitudes in $\vec{n}+{ }^{4} \mathrm{He}$ is almost orthogonal to the existing constraints from past measurements and the addition of $\vec{n}+{ }^{4} \mathrm{He}$ gives strong constraints [30].

Recently, an alternative to the DDH potential has been developed based on pionless effective field theory (EFT) [31-33]. The advantages of this approach is that EFT treatment is model independent and has quantifiable errors and direct connection to QCD. When applying the $1 / N_{c}$ expansion of QCD [34,35], which is known to work at the $\sim 10 \%$ level for deriving the relative strengths of the NN strong interacting couplings, the implications were very surprising. The first conclusion was that the isovector NN parity violation amplitudes are small compared to both isoscalar and isotensor $\mathrm{NN}$ weak interactions. The other implication was that the commonly used isoscalar/isovector plot to display constraints of NN weak interactions is very deceiving as it is hiding a dominant contribution from the isotensor amplitude [11].

In Ref. [11], Gardner, Holstein and Haxton have for the first time reorganized the theory analysis to take into account the recent theory results from the QCD $1 / N_{c}$ expansion, which gives a natural scale for the relative size of the weak couplings. It leads to a completely different picture of the NN weak interaction. In particular, it has very important positive implications for the scientific impact of $\vec{n}+{ }^{4} \mathrm{He}$ spin rotation. The theory is now in a position to predict $\vec{n}+{ }^{4} \mathrm{He}$ neutron spin rotation from the Standard Model. The predicted size of the spin rotation angle is large, $6.8 \times 10^{-7} \mathrm{rad} / \mathrm{m}$, which is based on the assumption that the three $\mathrm{N}^{2} \mathrm{LO}$ low-energy constants (LECs) are negligible [11]. However, very recent NPDGamma results [29] show that the $\mathrm{N}^{2} \mathrm{LO}$ LECs can not be neglected. Including the $\mathrm{N}^{2} \mathrm{LO}$ LECs and NPDGamma results [29] in the EFT+leading- $\mathrm{N}_{c}$ expansion gives a neutron spin rotation angle in ${ }^{4} \mathrm{He}$ of $(9.0 \pm 1.5) \times 10^{-7} \mathrm{rad} / \mathrm{m}[11,36]$. The statistical error of the earlier version of the $\vec{n}+{ }^{4} \mathrm{He}$ experiment was $9 \times$ $10^{-7} \mathrm{rad} / \mathrm{m}$ [4], and with the new very intense beam of cold neutrons at NIST we should be able to reach a statistical accuracy of better than $\sim 1 \times 10^{-7} \mathrm{rad} / \mathrm{m}$.

The $\vec{n}+{ }^{3}$ He experiment is now completed at SNS and a final result with a very small value was released at the APS DNP2017 meeting. The $\vec{n}+{ }^{3} \mathrm{He}$ collaboration is still investigating some of the associated systematic uncertainties. The $\vec{n}+{ }^{3} \mathrm{He}$ experiment is sensitive to the isotensor component of the NN weak interaction, whereas $\vec{n}+{ }^{4} \mathrm{He}$ is not. Since the isotensor NN weak amplitude is now understood from theoretical considerations to be one of the two leading-order terms in $\mathrm{NN}$ weak interaction, this is an important distinction between the two experiments.

In $\vec{n}+{ }^{4} \mathrm{He}$, the NSR collaboration used parity violating spin rotation in liquid ${ }^{4} \mathrm{He}$ to constrain hadronic weak coupling constants and measured a neutron spin rotation angle per unit length $(d \phi / d z)$ of $[+1.7 \pm$ 9.1 (stat.) \pm 1.4 (sys.) $] \times 10^{-7} \mathrm{rad} / \mathrm{m} \quad$ [4]. Given the recent EFT+leading- $\mathrm{N}_{c}$ calculations of $d \phi / d z[11,36]$, an improvement on the statistical uncertainties to the level of $1 \times 10^{-7}$ will be of a great value. At a minimum it would suffice to place a tight constraint on NN weak interaction amplitudes but can become the first test of the Standard Model in the NN weak sector if a nonzero value obtained as predicted by theory.

The phenomenon of parity-violating neutron spin rotation in $\vec{n}+{ }^{4} \mathrm{He}$ can be understood in terms of neutron optics. The parity-violating $(P V)$ weak interaction causes the amplitudes of the positive and negative neutron helicity states moving through a medium to accumulate different phases. The difference $\phi_{P V}$ between the phase shifts of the helicity states leads to a rotation of the neutron polarization about its momentum, which manifestly violates parity [37]. The rotation angle per unit length is proportional to the forward limit of the parity-odd $p$-wave scattering amplitude $f_{P V}, d \phi / d z=4 \pi \rho f_{P V} / k$. Because $f_{P V}$ is proportional to the parity-odd correlation $\vec{\sigma}_{n} \cdot \vec{k}_{n}$ with $\vec{\sigma}_{n}$ the neutron spin vector and $\vec{k}_{n}$ the neutron momentum, it tends to a constant for low energy neutrons in the absence of resonances [38].

\section{Experimental setup}

Measuring a very small spin-rotation angle of $10^{-7} \mathrm{rad} / \mathrm{m}$ is very challenging and requires an apparatus that distinguishes the small parity-violating rotations from rotations that arise from residual magnetic fields. The apparatus consists of an upgraded version of that used in the previous NSR measurement [40-42]. The old apparatus consisted of a neutron supermirror polarizer, an adiabatic RF spin-flipper, nonmagnetic neutron guides, spin transport, an adiabatic spin rotator, a neutron supermirror polarization analyzer, and a current-mode ion chamber [41,42]. Figure 1 shows a diagram of the neutron beam-line and path along the spin rotation apparatus. Details of the measurement technique and the apparatus can be found elsewhere [39]. 


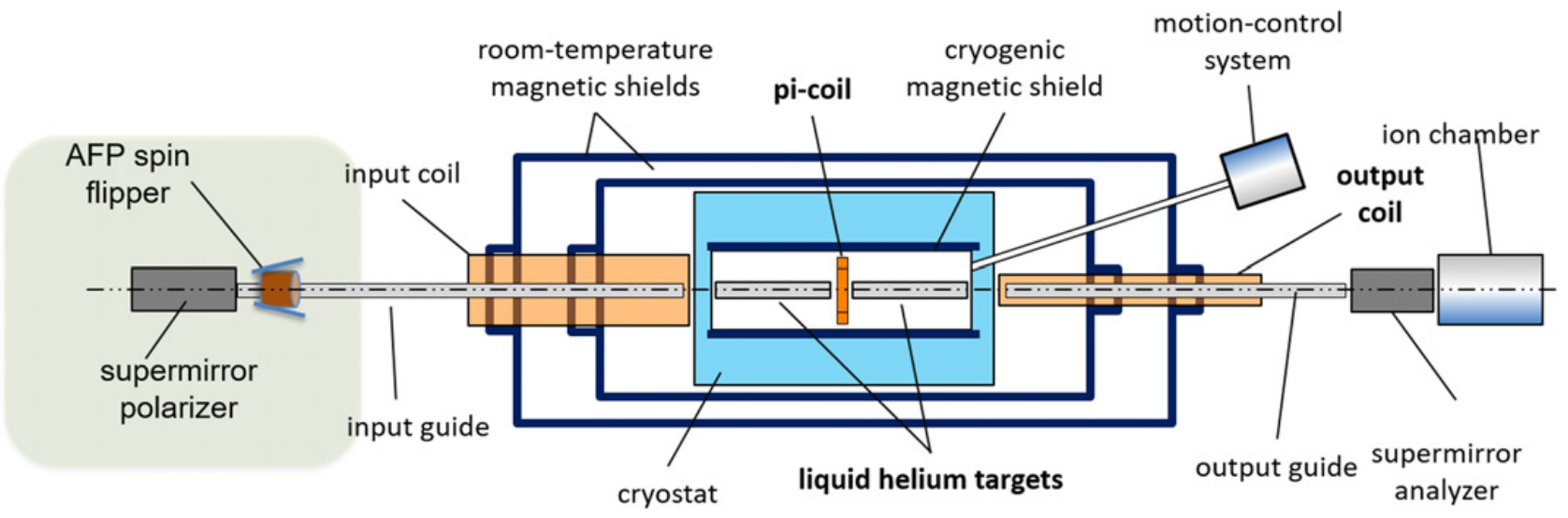

Figure 1. Top view diagram of the spin rotation apparatus [39]. The target consists of four chambers and a $\pi$-coil.

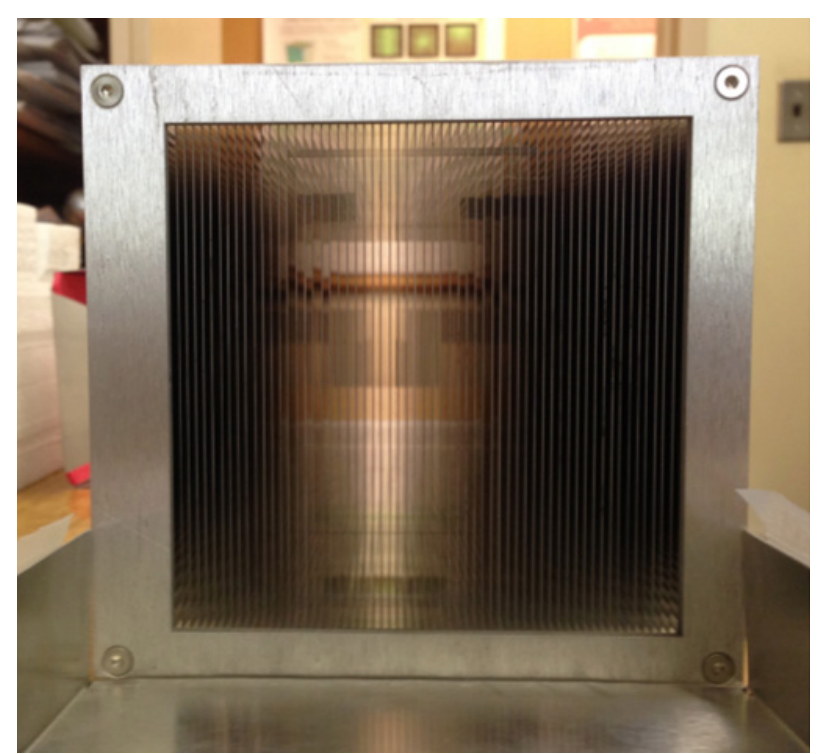

Figure 2. Front view of the SM. The image seen through the SM is deflected by $13 \mathrm{mrad}$.

\section{Toward an improved measurement}

The recent high intensity neutron beam (NG-C) at NIST [43] has a factor of 20 increase on NG-6 flux which provides the opportunity to drastically improve the statistically limited previous NSR measurement [4]. However, NG-C has larger cross sectional area $(11 \times$ $\left.11 \mathrm{~cm}^{2}\right)$ and larger divergence $(5.95 \mathrm{mrad} / \AA)$ than the NG-6 neutron beam with $6 \times 15 \mathrm{~cm}^{2}$ cross sectional area and $2.0 \mathrm{mrad} / \AA \AA$ divergence which necessitated upgrading the NSR apparatus to take advantage of the larger-area and more divergent NG-C beam.

In addition, several other improvements on NSR setup were carried out. These improvements include two new $10 \times 10 \mathrm{~cm}^{2}$ polarizing super-mirrors with $60 \mathrm{Fe} / \mathrm{Si} m=$ 2.5 super-mirror ( $\mathrm{SM}$ ) blades to polarize and analyze the beam. The SM polarizer is shown in Fig. 2. These SM benders have a transmission of greater than $90 \%$ for one spin state and a transmission of less than $0.5 \%$ for the other spin state.

To retain a greater fraction of the more divergent beam on NG-C, new $10 \times 10 \mathrm{~cm}^{2}$ with $m=2$ non-magnetic (NiMo/Ti) super-mirror waveguides were constructed. The new input and output guides have $<1 \%$ depolarization

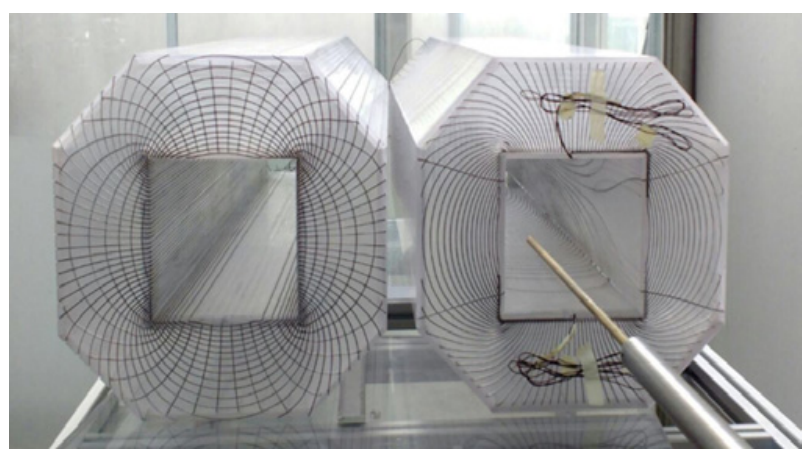

Figure 3. Front view of the input and output coils, each with two layers of acrylic, one for each field component (vertical and transverse). The output coil (left) has two layers of windings: one to produce the transverse decreasing field, which also needs to alternate polarity to produce clockwise or counterclockwise rotations of the neutron spin; and the second to produce a vertical increasing field [44].

probability per bounce. New input and output coils were built to match the larger guides. The coils consist of wires woven into grooves etched into hollow hexagonal plastic extrusions with a rectangular cavity in the center to fit the SM guides [44], as shown in Fig. 3.

One of the main challenges during the previous NSR experiment was moving and maintaining liquid ${ }^{4} \mathrm{He}$ in the cryostat, and more than $25 \%$ of the beam-on time was lost in this process. Therefore, more efficient liquid ${ }^{4} \mathrm{He}$ pumping system that includes a ${ }^{4} \mathrm{He}$ liquefier was built. The ${ }^{4} \mathrm{He}$ liquefier is expected to operate continuously by converting helium boil-off gas from the helium cryostat to liquid helium and delivering liquid helium back into the cryostat. The ${ }^{4} \mathrm{He}$ liquefier was tested for three months of continuous operation at an observed liquefaction rate from warm gas of 12 liters per day. With automated operation capable of handling $\sim 550 \mathrm{~mW}$ heat load, the ${ }^{4} \mathrm{He}$ liquefier will also reduce down time by improving the management of the liquid ${ }^{4} \mathrm{He}$ target. An enlarged ${ }^{4} \mathrm{He}$ target is being constructed to match the cross sectional area of the NG-C beam and fit with the new ${ }^{4} \mathrm{He}$ pumping system. Figure 4 shows the design of the apparatus. All of the target components including the cryostat and ${ }^{4} \mathrm{He}$ liquefier are constructed and commissioned, and we are currently testing the ${ }^{4} \mathrm{He}$ pump. 
Table 1. Summary of the upgraded and new components for NSR experiment on NG-C compared with those of NSR on NG-6.

\begin{tabular}{lcc}
\hline \hline & NSR on NG-6 & NSR on NG-C \\
\hline Thermal equivalent flux & $4.5 \times 10^{8} \mathrm{~cm}^{-2} \mathrm{~s}^{-1}$ & $8 \times 10^{9} \mathrm{~cm}^{-2} \mathrm{~s}^{-1}$ \\
Polarizer/Analyzer & & New $/ \mathrm{m}=2.5$ \\
Cross sectional area & $5 \times 5 \mathrm{~cm}^{2}$ & $10 \times 10 \mathrm{~cm}^{2}$ \\
Input/output guides & $m=0.68$ & $m=2$ \\
Background field & $100 \mu \mathrm{G}$ & $10 \mu \mathrm{G}$ \\
\hline Uncertainties & 9.1 (stat) \pm 1.4 (sys) & 1.0 (stat) \pm 1.0 (sys) \\
\hline \hline
\end{tabular}

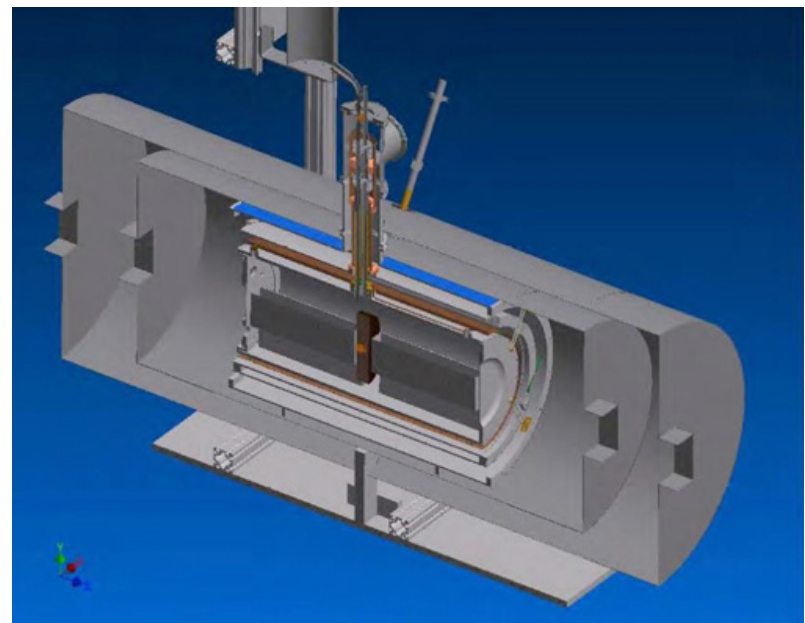

Figure 4. Cross sectional design view of the ${ }^{4} \mathrm{He}$ target region with a port through the top to the He liquefier.

A new $10 \mathrm{~cm}$ diameter ion chamber of similar design to the existing chamber was constructed. Additional improvements on the magnetic shielding in the target region were carried out to maintain magnetic fields below $10 \mu \mathrm{G}$ in the target region which keeps the associated systematic uncertainties at $\sim 1.0 \times 10^{-7}$. Calculations of the involved systematic uncertainties are discussed elsewhere [39]. A summary of the improvements related to the new NSR experiment is shown in Table 1. With the higher NG-C flux and various upgraded experiment components, we expect the new experiment to reach statistical and systematic precision of $[1.0$ (stat) \pm 1.0 (sys) $] \times 10^{-7} \mathrm{rad} / \mathrm{m}$ for the extracted parity violating spin rotation angle.

All of the NSR components except for the ${ }^{4} \mathrm{He}$ target were constructed and then commissioned at LANSCE on FP12 beam-line. They were used for another measurement, that is discussed in Sect. 4, and performed as intended.

\section{Search for possible exotic spin dependent interactions using NSR apparatus}

The NSR apparatus was also used to search for a possible new axial vector interaction in the millimeter to micron range using spin dependent interactions of neutrons with matter though exchange of spin 1 bosons as predicted in some extensions of the Standard Model. This experiment was performed on FP12 at the LANSCE facility at Los Alamos by passing transversely polarized slow neutrons through gaps between slabs of copper

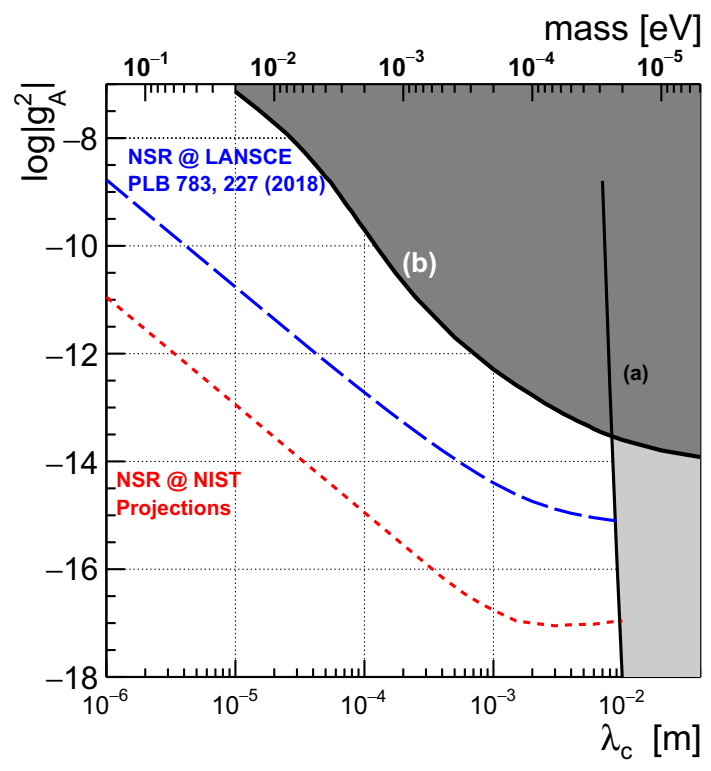

Figure 5. Projections of $g_{A}^{2}$ as a function of $\lambda_{c}$ from the proposed measurement at NG-C (dashed-red) compared with constraints from LANSCE measurement (longdashed-blue) [46], $\mathrm{K}-{ }^{3} \mathrm{He}$ comagnetometry (a) [47] and from a neutron measurement using Ramsey spectroscopy (b) [48].

and float glass arranged so that the possible exotic interaction would tilt the plane of polarization along the neutron momentum [45]. The resulting rotation angle, $\phi=$ $[+2.8 \pm 4.6$ (stat.) \pm 4.0 (sys.) $] \times 10^{-5} \mathrm{rad} / \mathrm{m}$, is consistent with zero [46]. This constraint improves on the previous upper bounds on $g_{A}^{2}$ by about three orders of magnitude for $\lambda_{c}$ between $1 \mathrm{~cm}$ and $1 \mu \mathrm{m}$.

This result was obtained with a week worth of data at LANSCE and the NG-C beam provides the opportunity to drastically improve on these constraints. In addition, we are currently upgrading the room temperature target by using tungsten plates instead of copper plates to further increase mass density gradient in target which further enhances the studied effect. With one calendar month of beam time on NG-C and with the upgraded target we expect to set a new limit on possible exotic axial vector couplings of the neutron to matter which is two to three orders of magnitude more stringent than the previous measurement at LANSCE [46], as shown in Fig. 5.

\section{Conclusion}

Significant recent theoretical work predicted a relatively large neutron spin rotation, $(9.0 \pm 1.5) \times 10^{-7} \mathrm{rad} / \mathrm{m}$, in ${ }^{4} \mathrm{He}$ without sensitivity to the isotensor component of the 
NN weak interaction, a strong distinction between $\vec{n}+{ }^{3} \mathrm{He}$ and $\vec{n}+{ }^{4} \mathrm{He}$. The NSR collaboration substantially improved the previous NSR apparatus to repeat the measurement at the high flux NG-C beam-line. This is expected to yield a measurement at the level of $[ \pm 1.0$ (stat.) \pm 1.0 (sys.) $] \times$ $10^{-7} \mathrm{rad} / \mathrm{m}$ which would provide the first test of the Standard Model in the NN weak sector.

The upgraded apparatus was used to make significant improvement in limits on spin-dependent fifth forces using a room temperature target. This constraint improves on the previous upper bounds on $g_{A}^{2}$ by 2-4 orders of magnitude for $\lambda_{c}$ between $1 \mathrm{~cm}$ and $1 \mu \mathrm{m}$. Running the upgraded room temperature target with tungsten plates on NG-C beam line for a calendar month is expected to constrain $g_{A}^{2}$ by at least two additional orders of magnitude in this range of $\lambda_{c}$.

The work of M. Sarsour was supported by the US Department of Energy grant DE-SC0010443. E. Anderson, D. Esposito, I. Francis, K. Korsak, W. M. Snow, K. Steffen, and J. Vanderwerp acknowledge support from NSF PHY-1614545 and from the Indiana University Center for Spacetime and Symmetries. We acknowledge the support of the National Institute of Standards and Technology, US Department of Commerce, in providing some of the neutron facilities used in this work.

\section{References}

[1] J. Erler, M. Ramsey-Musolf, Prog. Part. Nucl. Phys. 54, $351(2005)$

[2] M.J. Ramsey-Musolf, S.A. Page, Ann. Rev. Nucl. Part. Sci. 56, 1 (2006)

[3] W.M. Snow, Eur. Phys. J. A 24, 119 (2005)

[4] W.M. Snow et al., Phys. Rev. C 83, 022501 (2011)

[5] C.S. Wood, S.C. Bennett, D. Cho, B.P. Masterson, J.L. Roberts, C.E. Tanner, C.E. Wieman, Science 275, 1759 (1997)

[6] Y.B. Zeldovich, Sov. Phys. JETP 6, 1184 (1957)

[7] V.V. Flambaum, I.B. Khriplovich, Sov. Phys. JETP 52, 835 (1980)

[8] S. Tomsovic, M.B. Johnson, A.C. Hayes, J.D. Bowman, Phys. Rev. C 62, 054607 (2000)

[9] B. Desplanques, J.F. Donoghue, B.R. Holstein, Annals Phys. 124, 449 (1980)

[10] E.G. Adelberger, W.C. Haxon, Ann. Rev. Nucl. Part. Sci. 35, 501 (1985)

[11] S. Gardner, W.C. Haxon, B.R. Holstein, Ann. Rev. Nucl. Part. Sci. 67, 69 (2017)

[12] P. Eversheim et al., Phys. Lett. B 256, 11 (1991)

[13] R. Balzer et al., Phys. Rev. Lett. 44, 699 (1980)

[14] R. Balzer et al., Phys. Rev. C 30, 1409 (1984)

[15] S. Kistryn et al., Phys. Rev. Lett. 58, 1616 (1987)

[16] A.R. Berdoz et al., Phys. Rev. C 68, 034004 (2003)

[17] A.R. Berdoz et al., 87, 272301 (2001)
[18] J. Lang et al., Phys. Rev. Lett. 54, 170 (1985)

[19] R. Henneck et al., Phys. Rev. Lett. 48, 725 (1982)

[20] C.A. Barnes et al., Phys. Rev. Lett. 40, 840 (1978)

[21] G. Ahrens et al., Nucl. Phys. A 390, 486 (1982)

[22] M. Bini, T.F. Fazzini, G. Poggi, N. Taccetti, Phys. Rev. Lett. 55, 795 (1985)

[23] S.A. Page et al., Phys. Rev. C 35, 1119 (1987)

[24] E.G. Adelberger et al., Phys. Rev. C 27, 2833 (1983)

[25] K. Elsener et al., Nucl. Phys. A 461, 579 (1987)

[26] K. Elsener et al., Phys. Rev. Lett. 52, 1476 (1984)

[27] W.S. Wilburn, J.D. Bowman, Phys. Rev. C 57, 3425 (1998)

[28] M.M. Musgrave et al., Nucl. Instrum. Methods Phys. Res., Sect. A 895, 19 (2018)

[29] D. Blyth, J. Fry, N. Fomin, R. Alarcon, L. Alonzi, E. Askanazi, S. Baeßler, S. Balascuta, L. BarrónPalos, A. Barzilov et al. (NPDGamma Collaboration), Phys. Rev. Lett. 121, 242002 (2018)

[30] V. Dmitriev, V. Flambaum, O. Sushkov, V. Telitsin, Phys. Lett. B 125, 1 (1983)

[31] S.L. Zhu, C. Maekawa, B. Holstein, M. RamseyMusolf, U. van Kolck, Nucl. Phys. A 748, 435 (2005)

[32] L. Girlanda, Phys. Rev. C 77, 067001 (2008)

[33] D.R. Phillips, M.R. Schindler, R.P. Springer, Nucl. Phys. A 822, 1 (2009)

[34] D.R. Phillips, D. Samart, C. Schat, Phys. Rev. Lett. 114, 062301 (2015)

[35] M.R. Schindler, R.P. Springer, J. Vanasse, Phys. Rev. C 93, 025502 (2016)

[36] W.M. Snow, Private Communication (2018)

[37] F.C. Michel, Phys. Rev. 133, B329 (1964)

[38] L. Stodolsky, Nucl. Phys. B 197, 213 (1982)

[39] W.M. Snow et al., Rev. Sci. Instrum. 86, 055101 (2015)

[40] W. Snow, Nucl. Instrum. Methods Phys. Res., Sect. A 611, 248 (2009)

[41] C. Bass et al., Nucl. Instrum. Methods Phys. Res., Sect. A 612, 69 (2009)

[42] A. Micherdzinska et al., Nucl. Instrum. Methods Phys. Res., Sect. A 631, 80 (2011)

[43] J.C. Cook, Rev. Sci. Instrum. 80, 023101 (2009)

[44] Maldonado-Velázquez, Barrón-Palos, C. Crawford, W. Snow, Nucl. Instrum. Methods Phys. Res., Sect. A 854, 127 (2017)

[45] C. Haddock et al., Nucl. Instrum. Methods Phys. Res., Sect. A 885, 105 (2018)

[46] C. Haddock et al., Phys. Lett. B 783, 227 (2018)

[47] G. Vasilakis, J.M. Brown, T.W. Kornack, M.V. Romalis, Phys. Rev. Lett. 103, 261801 (2009)

[48] F.M. Piegsa, G. Pignol, Phys. Rev. Lett. 108, 181801 (2012) 